\title{
On Cosmological Implication of the Trace Anomaly
}

\author{
Y. Bisabr* \\ Department of Physics, Shahid Rajaee University, Lavizan, Tehran 16788, Iran.
}

\begin{abstract}
We establish a connection between the trace anomaly and a thermal radiation in the context of the standard cosmology. This is done by solving the covariant conservation equation of the stress tensor associated with a conformally invariant quantum scalar field. The solution corresponds to a thermal radiation with a temperature which is given in terms of a cut-off time excluding the spacetime regions very close to the initial singularity. We discuss the interrelation between this result and the result obtained in a two-dimensional schwarzschild spacetime.
\end{abstract}

\section{Introduction}

One of the important approaches to quantum gravity concerns a framework in which matter is described by quantum field theory while the gravitational field itself is regarded as a classical object. In this framework, the so-called quantum field theory in curved spacetime, the stress tensor associated with a quantum field is not well-defined and contains singularities. Thus, some renormalization prescriptions [1] are usually used to obtain a meaningful expression for the stress tensor of a quantum field. One of the most remarkable consequences of these prescriptions is the so-called trace anomaly [2]. This means that the trace of the quantum stress tensor of a conformal invariant field obtains a nonzero expression while the trace of the classical stress tensor vanishes identically.

It is shown [3] that in a two-dimensional schwarzschild spacetime there is a close correspondence between the trace anomaly and Hawking radiation [4], namely the thermal radiation associated with a black hole at null infinity. The radiation has a temperature $T=\left(4 \pi k_{B} R_{S}\right)^{-1}$ where $k_{B}$ is the Boltzmann's constant and $R_{S}$ is the schwarzschild radius of a black hole. It should be noted that the length scale $R_{S}$ may be interpreted as a cut-off length excluding the intrinsic singularity in the interior region of the schwarzshcild solution. In this sense, the

*e-mail: y-bisabr@srttu.edu. 
temperature of the Hawking radiation is given in terms of a cut-off length, $R_{S}$, characteristic to the schwarzshcild spacetime.

The basic question we wish to address in the present work is that whether there exists such a correspondence between the trace anomaly and a thermal radiation in a cosmological context. To address this question we note that the trace anomaly is a consequence of quantum behaviour of conformal invariant matter systems, the characteristic of the matter systems at sufficiently early times in the standard cosmology. Following the result obtained in the schwarzschild spacetime that the trace anomaly has a long range effect at null infinity, one can also expect here that it is possible to relate trace anomaly to properties of a thermal radiation at late-time evolution of the universe.

Here we intend to establish such a relation in two parts. In section 2, we first use a twodimensional cosmological model to find the most general solution of the covariant conservation equation of the quantum stress tensor associated with a conformally invariant scalar field. We shall show that the solution corresponds to an equilibrium gas with a temperature $T \propto\left(k_{B} t_{c}^{-1}\right)$ with $t_{c}$ being a cut-off time. This cut-off time is defined to avoid the spacetime regions in which the semiclassical investigations do not valid. In section 3, we shall show that contrary to the schwarzschild spacetime, the symmetries of the standard cosmology allow us to solve the covariant conservation equation in four dimensions. We solve the conservation equations for spatially flat Friedmann-Robertson-Walker spacetime. At late times, the solution corresponds to a thermal radiation with a temperature of the same order of the temperature obtained in the two-dimensional case. In section 4, we outline our results.

Throughout the following we shall use units in which $\hbar=c=1$ and the signature is $(-+++)$.

\section{The Model}

Let us begin with the results of renormalization of stress tensor $T_{\mu}^{\nu}$ of a quantum scalar field coupled with a two-dimensional gravitational background

$$
\begin{gathered}
\nabla_{\nu} T_{\mu}^{\nu}=0 \\
T_{\mu}^{\mu}=\frac{1}{24 \pi} R
\end{gathered}
$$

where $\nabla_{\nu}$ denotes a covariant differentiation and $R$ is the curvature scalar. The first equation is a covariant conservation law and the second one indicates an anomalous trace emerging from the renormalization process.

We first intend to apply the conservation equation (1) to a two-dimensional cosmological model described by the metric

$$
d s^{2}=-d t^{2}+a^{2}(t) d x^{2}
$$

where $a(t)$ is the scale factor. This is a two-dimensional analog of the spatially flat FriedmannRobertson-Walker (FRW) spacetime. The metric (3) can be written in a conformally flat form 


$$
d s^{2}=a^{2}(\tau)\left(-d \tau^{2}+d x^{2}\right)
$$

where

$$
\tau=\int \frac{d t}{a(t)}
$$

is the conformal time. For the metric (4), the nonvanishing Christoffel symbols are given by

$$
\Gamma_{\tau \tau}^{\tau}=\Gamma_{x x}^{\tau}=\Gamma_{\tau x}^{x}=\frac{1}{a} \frac{d a}{d \tau}
$$

In the spacetime described by (4), all components of the stress tensor can be only functions of time. Using this fact, the conservation equation (1) takes the form

$$
\begin{aligned}
& \frac{d}{d \tau} T_{\tau}^{\tau}+\Gamma_{x \tau}^{x} T_{\tau}^{\tau}-\Gamma_{x \tau}^{x} T_{x}^{x}=0 \\
& \frac{d}{d \tau} T_{x}^{\tau}+\Gamma_{\tau \tau}^{\tau} T_{x}^{\tau}-\Gamma_{x x}^{\tau} T_{\tau}^{x}=0
\end{aligned}
$$

For the off-diagonal elements of the stress tensor we have $T_{x}^{\tau}=-T_{\tau}^{x}$. On the other hand, one can write $T_{x}^{x}=T_{\mu}^{\mu}-T_{\tau}^{\tau}$. These relations among different components of the stress tensor together with (5) and (6) allow us to write (7) and (8) in the form

$$
\begin{gathered}
\frac{d}{d t}\left(a^{2} T_{\tau}^{\tau}\right)=a \frac{d a}{d t} T_{\mu}^{\mu} \\
\frac{d}{d t}\left(a^{2} T_{x}^{\tau}\right)=0
\end{gathered}
$$

The equation (10) gives immediately

$$
T_{x}^{\tau}=\alpha a^{-2}
$$

with $\alpha$ being an integration constant. The solution of the equation (9) is

$$
T_{\tau}^{\tau}=a^{-2}(h+\beta)
$$

where

$$
\begin{gathered}
h=\int_{t_{c}}^{t} T_{\mu}^{\mu}\left(t^{\prime}\right) \frac{d a\left(t^{\prime}\right)}{d t^{\prime}} a\left(t^{\prime}\right) d t^{\prime} \\
\beta=a^{2}\left(t_{c}\right) T_{\tau}^{\tau}\left(t_{c}\right)
\end{gathered}
$$

and $t_{c}$ is an arbitrary time scale. Given a time scale $t_{c}$, the function $h$ incorporates the corresponding contribution of the trace $T_{\mu}^{\mu}$ in the stress tensor $T_{\mu}^{\nu}$. The introduction of the cut-off time $t_{c}$ is a mandate in order to exclude in the definition of $h$ the contribution of the trace very close to the early time singularity. In fact in that region an accurate description of quantum gravity is needed and the semiclassical approach is no longer valid. 
In the context of the cosmology described by (4), we require that $\alpha=0$. This implies that the stress tensor has vanishing off-diagonal elements. In this case we obtain

$$
T_{\mu}^{\nu}=a^{-2}(h+\beta)\left(\begin{array}{cc}
1 & 0 \\
0 & q-1
\end{array}\right)
$$

where

$$
q=\frac{T_{\mu}^{\mu}}{a^{-2}(h+\beta)}
$$

We are particularly interested in the late-time configuration of the stress tensor. It obviously depends on the explicit form of the scale factor. Thus for studying $T_{\mu}^{\nu}$ at late times, we first assume that the scale factor follows a power law expansion

$$
a=a_{0}\left(\frac{t}{t_{0}}\right)^{n}
$$

with $t_{0}$ being the present age of the universe. We then use the explicit form of the trace anomaly for the metric (4) and in terms of the scale factor (17) ( see the Appendix ) to write (13) and (16) in the form

$$
\begin{gathered}
h\left(t \rightarrow t_{0}\right)=\frac{n^{2} a_{0}^{2} t_{0}^{-2}}{24 \pi}\left\{1-l^{2(n-1)}\right\} \\
q\left(t \rightarrow t_{0}\right)=\frac{2(n-1)}{n}\left\{1+\frac{n-2}{n} l^{2(n-1)}\right\}^{-1}
\end{gathered}
$$

where $l=\frac{t_{c}}{t_{0}}$. Using (14) and (18), the stress tensor (15) takes the form

$$
T_{\mu}^{\nu}\left(t \rightarrow t_{0}\right)=\frac{n^{2} t_{0}^{-2}}{24 \pi}\left\{1+\frac{n-2}{n} l^{2(n-1)}\right\}\left(\begin{array}{cc}
1 & 0 \\
0 & q\left(t \rightarrow t_{0}\right)-1
\end{array}\right)
$$

One should note that the cut-off time $t_{c}$ is much smaller than $t_{0}$ so that $l \ll 1$. One therefore infers that $l^{2(n-1)} \gg 1$ for $n<1$. In this case, we may apply the approximation that $q(t \rightarrow$ $\left.t_{0}\right) \ll 1$ which reduces $(20)$ to

$$
T_{\mu}^{\nu}\left(t \rightarrow t_{0}\right)=\frac{n(2-n) l^{2 n} t_{c}^{-2}}{24 \pi}\left(\begin{array}{cc}
-1 & 0 \\
0 & 1
\end{array}\right)
$$

When one compares (21) with the stress tensor of an equilibrium gas, namely

$$
\frac{\pi}{6}\left(k_{B} T\right)^{2}\left(\begin{array}{cc}
-1 & 0 \\
0 & 1
\end{array}\right)
$$

one concludes that at late times the stress tensor $T_{\mu}^{\nu}$ describes an equilibrium gas with temperature

$$
T=\frac{1}{2 \pi} \sqrt{n(2-n)} l^{n}\left(k_{B} t_{c}\right)^{-1}
$$

It is interesting to compare (23) with the result obtained in the schwarzschild spacetime. In that case, the temperature of the thermal radiation is given in terms of $R_{S}^{-1}$. The schwarzschild 
radius $R_{S}$ may be interpreted as a cut-off length that disjoints the interior and the exterior of the schwarzschild solution. In principle, this is very similar to the case of FRW cosmology. In this case, the temperature of the equilibrium gas is given in terms of $t_{c}^{-1}$. Here the cut-off time $t_{c}$ excludes the early stages of evolution of the universe in which the semiclassical calculations can not be applied.

\section{The four-dimensional case}

In the standard cosmology, the universe is assumed to be isotropic in all points of spacetime. This is a larger symmetry with respect to the schwarzshcild spacetime that allows us to generalize our results obtained in the previous section to four dimensions. In a four-dimensional spacetime, the analog of the equation (2) is

$$
T_{\mu}^{\mu}=-2 v_{1}(x)
$$

where

$$
v_{1}(x)=\frac{1}{720}\left(\square R-R_{\mu \nu} R^{\mu \nu}+R_{\mu \nu \gamma \delta} R^{\mu \nu \gamma \delta}\right)
$$

Here $\square \equiv g^{\mu \nu} \nabla_{\mu} \nabla_{\nu}, R_{\mu \nu}$ and $R$ are the first and the second contraction of the Riemann curvature tensor $R_{\mu \nu \gamma \delta}$, respectively. We apply the conservation equation (1) to the spatially flat FRW metric described by

$$
d s^{2}=-d t^{2}+a^{2}(t)\left(d x^{2}+d y^{2}+d z^{2}\right)
$$

or in a conformally flat form

$$
d s^{2}=a^{2}(\tau)\left(-d \tau^{2}+d x^{2}+d y^{2}+d z^{2}\right)
$$

where the conformal time $\tau$ is given by (5). The nonzero Christoffel symbols of the metric (27) are

$$
\Gamma_{\tau \tau}^{\tau}=\Gamma_{i i}^{\tau}=\Gamma_{\tau i}^{i}=\frac{1}{a} \frac{d a}{d \tau}
$$

where $i$ is a collective index denoting three space components.

We intend to find the most general solution of (1) for the metric (27). To do this, one should

note that in this case all the components of the stress tensor $T_{\mu}^{\nu}$ are space independent and can be only functions of time. We use this fact to write the time-component of the conservation equation (1) as

$$
\frac{d}{d \tau} T_{\tau}^{\tau}+3 \Gamma_{i \tau}^{i} T_{\tau}^{\tau}-3 \Gamma_{i \tau}^{i} T_{i}^{i}=0
$$

There are also three space-components that all have the same form

$$
\frac{d}{d \tau} T_{i}^{\tau}+\Gamma_{\tau \tau}^{\tau} T_{i}^{\tau}+3 \Gamma_{i \tau}^{i} T_{i}^{\tau}-\Gamma_{\tau i}^{i} T_{i}^{\tau}-\Gamma_{i i}^{\tau} T_{\tau}^{i}=0
$$

Note that there is no summation rule in the relations (28), (29) and (30). In these relations, one should simply replace the index $i$ with each of the space components, namely $x, y$ and $z$. If we use (5) and (28), the equations (29) and (30) take the form 


$$
\begin{gathered}
\frac{d}{d t}\left(a^{4} T_{\tau}^{\tau}\right)=a^{3} \frac{d a}{d t} T_{\mu}^{\mu} \\
\frac{d}{d t}\left(a^{4} T_{i}^{\tau}\right)=0
\end{gathered}
$$

in which we have also taken into account that

$$
T_{i}^{i}=\frac{1}{3}\left(T_{\mu}^{\mu}-T_{\tau}^{\tau}\right)
$$

and $T_{\tau}^{i}=-T_{i}^{\tau}$. The equations (32) and (31) yield

$$
\begin{gathered}
T_{i}^{\tau}=\delta a^{-4} \\
T_{\tau}^{\tau}=a^{-4}(H+\gamma)
\end{gathered}
$$

where

$$
\begin{gathered}
H=\int_{t_{c}}^{t} T_{\mu}^{\mu}\left(t^{\prime}\right) \frac{d a\left(t^{\prime}\right)}{d t^{\prime}} a^{3}\left(t^{\prime}\right) d t^{\prime} \\
\gamma=a^{4}\left(t_{c}\right) T_{\tau}^{\tau}\left(t_{c}\right)
\end{gathered}
$$

and $\delta$ is an integration constant. The homogeneity and isotropy of the universe require that the only possibly nonzero components of the stress tensor $T_{\mu}^{\nu}$ are $T_{\tau}^{\tau}$ and $T_{i}^{i}$. Under this requirement and using the trace condition (33), we obtain

$$
T_{\mu}^{\nu}=a^{-4}(H+\gamma) \operatorname{diag}\left\{1, \quad \frac{1}{3}(Q-1), \quad \frac{1}{3}(Q-1), \quad \frac{1}{3}(Q-1)\right\}
$$

where

$$
Q=\frac{T_{\mu}^{\mu}}{a^{-4}(H+\gamma)}
$$

We can use the explicit form of the trace anomaly (24) for the metric (27) and in terms of the scale factor (17) ( see the Appendix ) to obtain $H$ and $Q$ in asymptotic times

$$
\begin{gathered}
H\left(t \rightarrow t_{0}\right)=\frac{\lambda a_{0}^{4} n t_{0}^{-4}}{4(n-1)}\left\{1-l^{4(n-1)}\right\} \\
Q\left(t \rightarrow t_{0}\right)=\frac{4(n-1)}{n}\left\{1+\frac{(3 n-4)}{n} l^{4(n-1)}\right\}^{-1}
\end{gathered}
$$

We apply the same approximation used in the two-dimensional case, namely that $l^{4(n-1)} \gg 1$ for $n<1$ which results in $Q\left(t \rightarrow t_{0}\right) \ll 1$. Within this approximation and using (37) and $(40)$, (38) reduces to

$$
T_{\mu}^{\nu}\left(t \rightarrow t_{0}\right)=-\frac{n(3 n-4)}{4(n-1)} \lambda l^{4 n} t_{c}^{-4} \operatorname{diag}\left\{-1, \frac{1}{3}, \frac{1}{3}, \frac{1}{3}\right\}
$$


This corresponds to the stress tensor of a radiation with energy density $\rho \propto l^{4 n} t_{c}^{-4}$. There is a specific relation between the energy density and temperature $\rho \propto\left(k_{B} T\right)^{4}$ if the radiation is thermally distributed [5]. Therefore the temperature of the radiation is given by $T \propto$ $l^{n}\left(k_{B} t_{c}\right)^{-1}$ which is of the same order of the result obtained in the two-dimensional case.

\section{Concluding Remarks}

We have investigated the stress tensor of a conformally invariant quantum scalar field in a homogeneous and isotropic cosmology. We have solved the covariant conservation equation of the stress tensor in two different cases. Firstly, in a two-dimensional cosmology we have shown that the conservation equation is consistent with existence of a thermal radiation at late times. Secondly, in a homogeneous and isotropic universe we have been able to generalize our results to a four-dimensional spacetime. In this case, the general form of the stress tensor at late times is given by the stress tensor corresponding to a thermal radiation. In both cases the temperature of the radiation is given in terms of a cut-off time $t_{c}$. The cut-off is introduced to exclude the effects of early-time cosmology, in which a full theory of quantum gravity holds, from our semiclassical calculations. This result is analogous to the result obtained in the two-dimensional schwarzschild spacetime in the sense that in the latter case the temperature is given in terms of a cut-off length $R_{S}$ characteristic to the schwarzschild solution.

\section{Appendix}

We consider two metric tensors $\bar{g}_{\mu \nu}$ and $g_{\mu \nu}$ which are conformally related, namely

$$
\bar{g}_{\mu \nu}=\Omega^{2} g_{\mu \nu}
$$

where $\Omega$ is a smooth dimensionless spacetime function. If $g_{\mu \nu}$ describes the Minkowski spacetime, $\eta_{\mu \nu}$, the metric $\bar{g}_{\mu \nu}$ is said to be conformally flat.

In a two-dimensional spacetime, any metric tensor can take a conformally flat form. In this case the curvature scalar is given by $[6]$

$$
\bar{R}=-2\left(\Omega^{-2} \square_{\eta} \ln \Omega\right)
$$

where $\square_{\eta}$ is the d'Alamberian operator in Minkowski spacetime. Substituting this into the relation (2) and noting the fact that $\Omega(x)=a(\tau)$, we obtain for the trace anomaly

$$
T_{\mu}^{\mu}\left(\bar{g}_{\mu \nu}\right)=\frac{1}{12 \pi} \frac{1}{a} \frac{d^{2} a}{d t^{2}}
$$

in which we have used (5) to express the derivative of the scale factor with respect to the coordinate time $t$. If one uses the explicit form of the scale factor (17) in the relation (45), one arrives at 


$$
T_{\mu}^{\mu}\left(\bar{g}_{\mu \nu}\right)=\frac{1}{12 \pi} n(n-1) t^{-2}
$$

In the four-dimensional case, the trace anomaly (24) for the metrics $g_{\mu \nu}$ and $\bar{g}_{\mu \nu}$ are related by $[7]$

$$
\begin{gathered}
T_{\mu}^{\mu}\left(\bar{g}_{\mu \nu}\right)=-\frac{1}{360} e^{4 \omega}\left\{\square R-R_{\mu \nu} R^{\mu \nu}+R_{\mu \nu \gamma \delta} R^{\mu \nu \gamma \delta}+2 R \square \omega+2 R_{; \gamma} \omega^{; \gamma}+6 \square(\square \omega)+8(\square \omega)^{2}\right. \\
\left.-8 \omega_{; \mu \nu} \omega^{; \mu \nu}-8 R_{\mu \nu} \omega^{; \mu} \omega^{; \nu}-8 \omega_{; \gamma} \omega^{; \gamma} \square \omega-16 \omega_{; \mu \nu} \omega^{; \mu} \omega^{; \nu}\right\}
\end{gathered}
$$

where $\omega=-\ln \Omega$. If $g_{\mu \nu}=\eta_{\mu \nu},(47)$ reduces to

$$
T_{\mu}^{\mu}\left(\bar{g}_{\mu \nu}\right)=-\frac{1}{180} e^{4 \omega}\left\{3 \square_{\eta}\left(\square_{\eta} \omega\right)+4\left(\square_{\eta} \omega\right)^{2}-4 \omega_{; \mu \nu} \omega^{; \mu \nu}-4 \omega_{; \gamma} \omega^{; \gamma} \square_{\eta} \omega-8 \omega_{; \mu \nu} \omega^{; \mu} \omega^{; \nu}\right\}
$$

We may use $\omega=-\ln a$ and (5) to write $T_{\mu}^{\mu}\left(\bar{g}_{\mu \nu}\right)$ in the form

$$
T_{\mu}^{\mu}\left(\bar{g}_{\mu \nu}\right)=\frac{1}{60}\left\{\frac{1}{a} \frac{d^{4} a}{d t^{4}}+\frac{1}{a^{2}} \frac{d^{2} a}{d t^{2}}+3 \frac{1}{a^{2}} \frac{d a}{d t} \frac{d^{3} a}{d t^{3}}-3 \frac{1}{a^{3}} \frac{d^{2} a}{d t^{2}}\left(\frac{d a}{d t}\right)^{2}\right\}
$$

With the scale factor (17), (49) is equivalent to

$$
T_{\mu}^{\mu}=\lambda t^{-4}
$$

with

$$
\lambda=\frac{1}{30} n(n-1)\left(n^{2}-6 n+3\right)
$$

\section{References}

[1] N. D. Birrell and P. C. W. Davies, Quantum fields in curved space (Cambridge University Press, Cambridge, England, 1982)

[2] R. M. Wald, Commun. Math. Phys. 54, 1 (1977)

R. M. Wald, Phys. Rev. D 17, 1477 (1978)

[3] S. M. Christensen and S. A. Fulling, Phys. Rev. D 15, 2088 (1977)

[4] S. W. Hawking, Commun. math. Phys. 43, 199 (1975)

[5] E. W. Kolb and M. S. Turner, The Early Universe (Addison-Wesley Publishing company, 1990)

[6] R. M. Wald, General Relativity (The University of Chicago Press, 1984)

[7] M. R. Brown, J. Math. Phys. (N.Y.) 25, 136 (1984) 